\title{
Characterization of a High Strain Composite Material
}

\author{
I. Maqueda * and S. Pellegrino ${ }^{\dagger}$ \\ California Institute of Technology, CA 91125 \\ J. M. Mejia-Ariza $\ddagger$ \\ L'Garde Inc., Tustin, CA 92780
}

\begin{abstract}
L'Garde has designed and developed a high-strain composite material consisting of carbon fibers embedded in a silicone matrix. The behavior of this material is significantly different from standard composites and the paper presents special test methods to measure the properties of this material. It is found that rule of mixtures estimates are quite accurate for the longitudinal moduli in tension and bending, but less accurate for compression. The Poisson's ratio prediction is also not accurate. Regarding the strength of the composite, it is found that conservative predictions of tensile and compressive strengths can be obtained respectively from the Weibull distribution of the strength of a single fiber combined with a simple bundle theory, and the elastic fiber microbuckling stress.
\end{abstract}

\section{Nomenclature}

$A_{f} \quad$ Fiber tow cross-sectional area

$d \quad$ Sample diameter

E $\quad$ Elastic modulus

$E_{1}, E_{1 f}, E_{m}$ Longitudinal modulus, fiber modulus, matrix modulus

$F_{1 t} \quad$ Tensile strength

$G_{m} \quad$ Matrix shear modulus

$I \quad$ Area moment of inertia of sample cross-section

$l \quad$ Sample length

$m \quad$ Parameter of Weibull distribution

$M, M_{\text {crit }} \quad$ Bending moment, critical bending moment

$n \quad$ Randomly generated number between 0 and 1

$N, N_{f} \quad$ Number of fibers, total number of fibers

$P \quad$ Probability of failure of a single fiber

$t \quad$ Sample thickness

$V, V_{0} \quad$ Fiber volume, reference fiber volume

$V_{f}, V_{m} \quad$ Fiber volume fraction, matrix volume fraction

$w \quad$ Sample width

$\kappa \quad$ Curvature

$\nu_{12}, \nu_{12_{f}}, \nu_{m}$ Poisson's ratio, fiber Poisson's ratio, matrix Poisson's ratio

$\rho \quad$ Density

$\rho_{f} \quad$ Fiber density

$\rho_{m} \quad$ Matrix density

$\sigma, \sigma_{0}, \sigma_{\text {crit }} \quad$ Stress, reference stress, critical stress for microbuckling

()$_{t h} \quad$ Theoretical estimate

\footnotetext{
*Graduate Student, Graduate Aerospace Laboratories, 1200 East California Boulevard, Mail Code 105-50, AIAA student member, Fulbright Scholar, e-mail: imaqueda@caltech.edu

$\dagger$ Joyce and Kent Kresa Professor of Aeronautics and Professor of Civil Engineering, Graduate Aerospace Laboratories, 1200 East California Boulevard, Mail Code 301-46, Fellow AIAA. e-mail: sergiop@caltech.edu

${ }^{\ddagger}$ Director of Advanced Programs, 15181 Woodlawn Ave., Member AIAA. e-mail: juan@lgarde.com
} 


\section{Introduction}

Many deployable space structures make use of mechanical hinges to allow parts of the structure to rotate while the structure is folded. An alternate approach is to use, instead of hinges, thin-walled shell structures (tape springs) to provide elastically foldable connections. In this case the stored strain energy in the tape springs provides the energy that deploys the structure, but when the structure is folded the curvature changes in the tape springs have to be limited to the elastic range. A third approach is to use a foldable composite material in which the matrix either softens above a certain temperature (memory matrix composites) or is very soft (e.g. silicone). Sheets of these materials can be deformed by forming sharp kinks with almost no damage, as elastic microbuckling of the fibers acts as a stress relief mechanism that prevents them from breaking ${ }^{1-3}$ and these materials can be used to connect different parts of a deployable structure to provide an almost equivalent function to a mechanical hinge.

L'Garde has designed and developed a composite material made of carbon fibers embedded in a silicone matrix. ${ }^{4}$ Manufacturing techniques that localize the high-strain capability in narrow regions of a structure, so that elastic fold lines are formed only in these regions, while the rest of the structure is made from a composite with a stiffer matrix such as epoxy, have also be developed by L'Garde. Figure 1 demonstrates the folding of a carbon composite lamina with dual-matrix construction, where the high-strain capability is localized in a Miura-ori pattern.

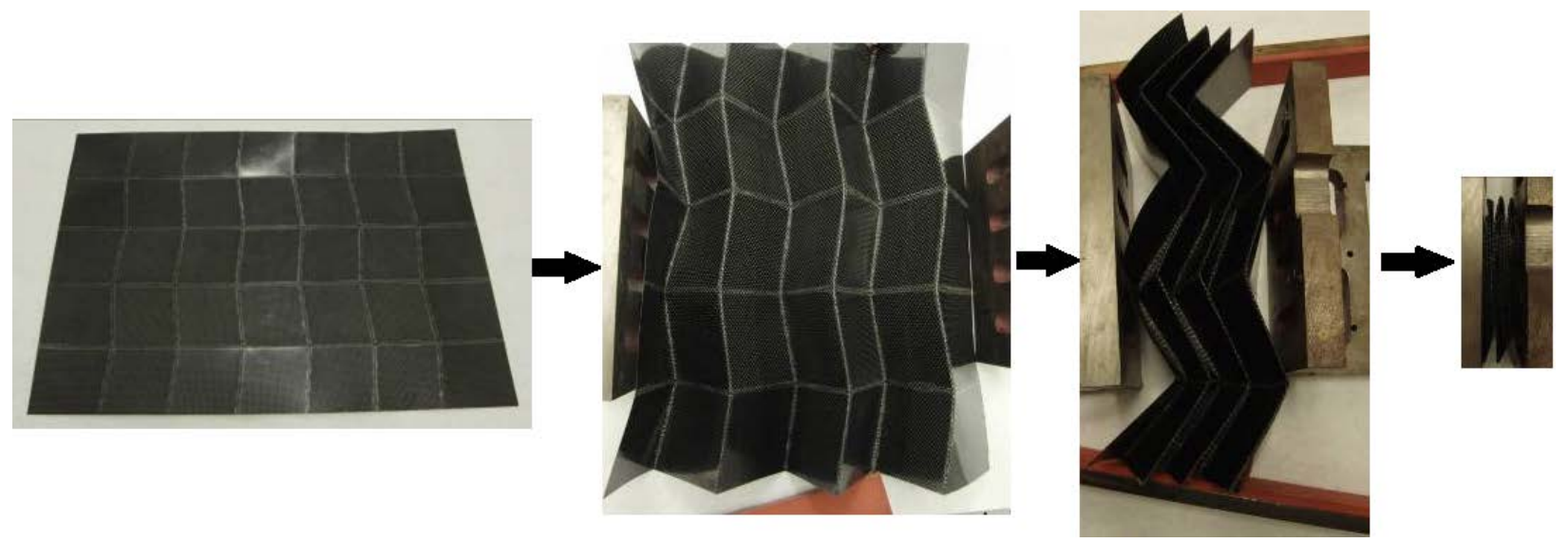

Figure 1. Folding sequence of a composite lamina in a Miura-Ori pattern.

Characterization of the mechanical properties of high strain composites requires the development of both analytical prediction techniques and novel experimental techniques that are suited to these materials. The present paper focuses mainly on experimental measurements of stiffness and strength, but also attempts some basic predictions in order to identify areas of future research.

The paper is organized as follows. Section II describes the large-strain material and the properties and geometry of the test samples. Section III presents preliminary estimates of the stiffness and strength of the samples, both for tension and compression. The experimental techniques developed for the study are presented in Section IV and the test results are in Section V. Section VI concludes the paper.

\section{Test Samples}

The material under study is made of parallel AS4 carbon fibers in a silicone matrix and is manufactured by L'Garde Inc. The density, elastic modulus and tensile strength of fibers and matrix are listed in Table 1.

\begin{tabular}{|c|c|c|c|c|}
\hline Material & $\rho\left(\mathrm{g} / \mathrm{cm}^{-3}\right)$ & $\mathrm{E}(\mathrm{GPa})$ & Poisson's ratio $\nu_{12}$ & Tensile Strength $(\mathrm{GPa})$ \\
\hline Carbon Fibers & 1.79 & 231 & 0.2 & 4.433 \\
\hline Silicone Matrix & 1.09 & 0.003 & 0.5 & 0.005 \\
\hline
\end{tabular}

Table 1. Density and elastic properties of matrix and fibers (provided by the supplier).

All tests were conducted on unidirectional samples with different geometries and fiber volume fractions. 
A fiber volume fraction estimation was obtained via density ratios, from:

$$
\rho=V_{f} \rho_{f}+V_{m} \rho_{m}
$$

Analysis of micrographs of the samples, obtained with the technique presented in Ref. ${ }^{2}$ gave results in agreement with this estimation.
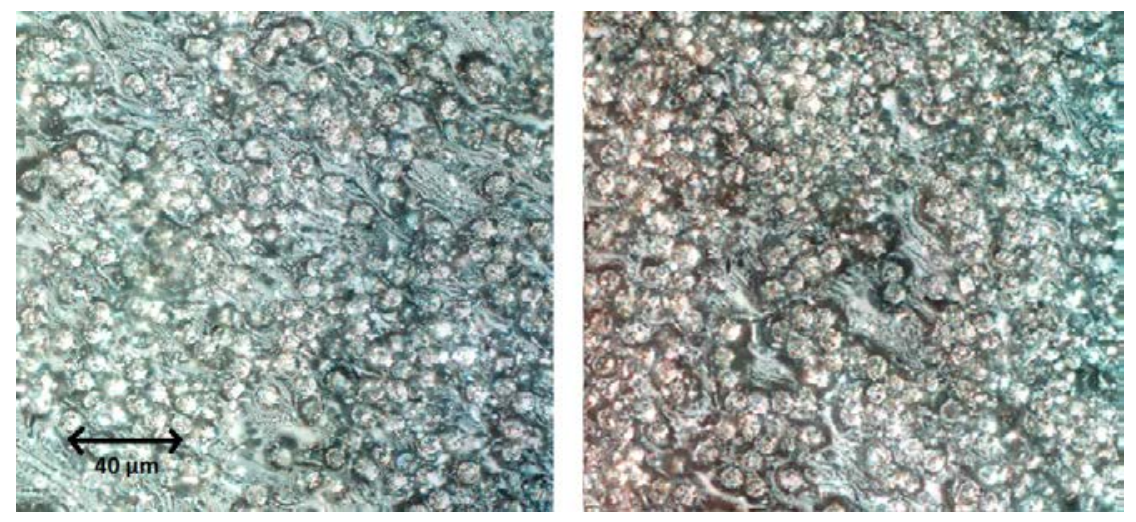

Figure 2. Micrographs of two different zones of the cross-section of a test sample.

Table 2 presents the main characteristics of the test samples, including their shape and size, as well as the theoretically estimated mechanical properties. The methods used to obtain these estimates are explained in the following section.

Flat unidirectional samples were used for the tension and bending tests, while for the compression tests unidirectional rods were chosen. $l$ is the sample length, $w$ the width, $t$ the thickness and $d$ the diameter. $E_{1}$ and $\nu_{12}$ are the estimated longitudinal stiffness and the Poisson's ratio of the case of longitudinal loading. The samples used for measuring the tensile strength were composed of a single tow of $N_{f}=12$, 000 fibers embedded in the soft matrix. The key parameter that defines this type of sample is the total cross-section of the fibers $A_{f}$.

\begin{tabular}{|c|c|c|c|c|}
\hline Test & Tension, Bending & Bending & Compression & Tensile Strength \\
\hline Shape & Flat & Flat & Rod & Tow \\
\hline Geometry $(\mathrm{mm})$ & $l=300 ; w=12 ; t=0.75$ & $l=300 ; w=12 ; t=1.5$ & $l=50 ; d=12$ & $l=200 ; A_{f}=0.48 \mathrm{~mm}^{2}$ \\
\hline$V_{f}(\%)$ & 33 & 35 & 47 & \\
\hline$E_{1}(\mathrm{GPa})$ & 78 & 83 & 108 & \\
\hline$\nu_{12}$ & & & 0.34 & \\
\hline$\sigma_{\text {crit }}(\mathrm{MPa})$ & 4.5 & 4.7 & 5.7 & \\
\hline$F_{1 t}(\mathrm{GPa})$ & & & & 1.5 \\
\hline
\end{tabular}

Table 2. Characteristics of test samples.

\section{Analytical Prediction of Material Properties}

\section{III.A. Stiffness}

The stiffness in the longitudinal direction can be estimated with the rule of mixtures:

$$
E_{1}=E_{1 f} V_{f}+E_{m} V_{m}
$$

Where $E_{1 f}$ is the longitudinal elastic modulus of the fibers, $E_{m}$ the Young's modulus of the matrix and $V_{f}$, $V_{m}$ are the fraction volume ratios of the fiber and matrix, respectively. The rule of mixtures has been used to estimate the Poisson's ratio $\nu_{12}$ as well.

$$
\nu_{12}=\nu_{12} V_{f}+\nu_{m} V_{m}
$$

These estimates have been calculated for the different samples tested and are presented in Table 2 . 


\section{III.B. Tensile strength}

The stiffness of the matrix is 5 orders of magnitude smaller than the stiffness of the fibers and hence it can be assumed that tensile loads are entirely carried by the fibers. The manufacturer's data for fiber strength, given in Table 1, typically corresponds to a probability of failure of $50 \%$ in a single fiber. Standard weak link theory was used to determine the behavior of a single fiber, ${ }^{5}$ leading to a Weibull distribution for the probability of failure

$$
P=1-\exp \left(\frac{-V}{V_{0}}\left(\frac{\sigma}{\sigma_{0}}\right)^{m}\right)
$$

where $V$ is the total volume of fiber, $\sigma$ is the stress, $V_{0}$ is the volume of fiber used to characterize the material and $\sigma_{0}$ is a reference stress for which a fiber of volume $V_{0}$ has a probability of failure $P=1-\exp (-1) \approx 0.0632$. The parameters of this distribution for these type of fibers have been determined in Ref. ${ }^{6}$ and the values obtained were $m=8.86$ and $\sigma_{0}=4.68 \mathrm{MPa}$.

The failure strength of a bundle of $N_{f}$ fibers, or tow, with total volume $V$, has been estimated using this probability of failure in a Monte Carlo simulation. The following process has been followed to perform the estimation:

1. Assuming the bundle of fibers is initially unstressed, a stress $\sigma=1 \mathrm{MPa}$ is applied and the current number of fibers, $N$, is set to $N_{f}$.

2. The probability of failure of one fiber in the bundle, $P$, is calculated according to Equation 4 and the following scheme is repeated:

(a) A random number $n$ between 0 and 1 is generated.

(b) If $n<P$, a fiber fails and the load in this fiber is reduced to zero. Then, $N=N-1$ and

- The stress in the bundle is recalculated to take into account the broken fiber: $\sigma=\sigma N /(N-1)$

- The effective volume is recalculated: $V=V(N-1) / N$

- The probability of failure of one fiber is recalculated with the new stress and volume.

- The process is restarted from step (a)

(c) If $n>P$, no fiber fails:

- The stress is increased by $1 \mathrm{MPa}: \sigma=\sigma+1$

- The probability of failure of one fiber is recalculated with the new stress.

- The process is restarted from step (a)

3. The process stops when the probability of failure of one fiber is $P=0.99$. This is the criterion for determining the failure stress in the bundle.

This process has been used with different values of the stress increment, yielding similar results. Due to the randomness involved, the process has been run several times, with no apparent variability in the resulting strength.

Figure 3 shows the fiber stress vs. the percentage of fibers broken in a $200 \mathrm{~mm}$ long tow of 12,000 carbon fibers. As it has been stated previously, the simulation was stopped at a probability that a fiber breaks in the tow of $99 \%$. The estimated strength of the tow is $1530 \mathrm{GPa}$, for which $22.4 \%$ of the fibers have failed.

\section{III.C. Compressive strength}

Fiber microbuckling has to be taken into account for both compression and bending tests. According to Rosen $^{7}$ under uniform compression the fibers can buckle in two possible modes: an extension mode where the fibers buckle out of phase, deforming the matrix in extension, and a shear mode where the fibers buckle in phase, shearing the matrix in between.

The shear mode is the less energetic one, thus it was considered for the estimation of the compression strength. Hence, the critical stress was calculated from

$$
\sigma_{c r i t}=\frac{G_{m}}{1-V_{f}}=\frac{E_{m}}{2\left(1-\nu_{m}\right)\left(1-V_{f}\right)}
$$




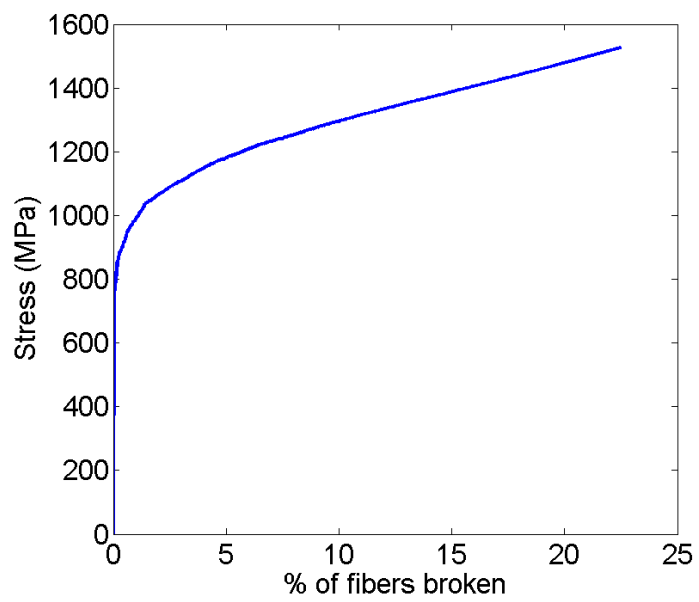

Figure 3. Prediction based on Monte Carlo simulation and weak link theory of behavior of a $200 \mathrm{~mm}$ long tow of 12,000 fibers.

\section{Test Configurations and Experimental Techniques}

\section{IV.A. Tension stiffness tests}

These tests, based on ASTM D3039, used the samples described in Table 2. They were gripped between Wyoming tensile wedge grips; emery cloth was wrapped around the clamped region of the sample to delay edge-slipping and the breaking of the fibers in the surface of the material due to the high compliance of the soft matrix. Figure 4 shows the experiment set up.
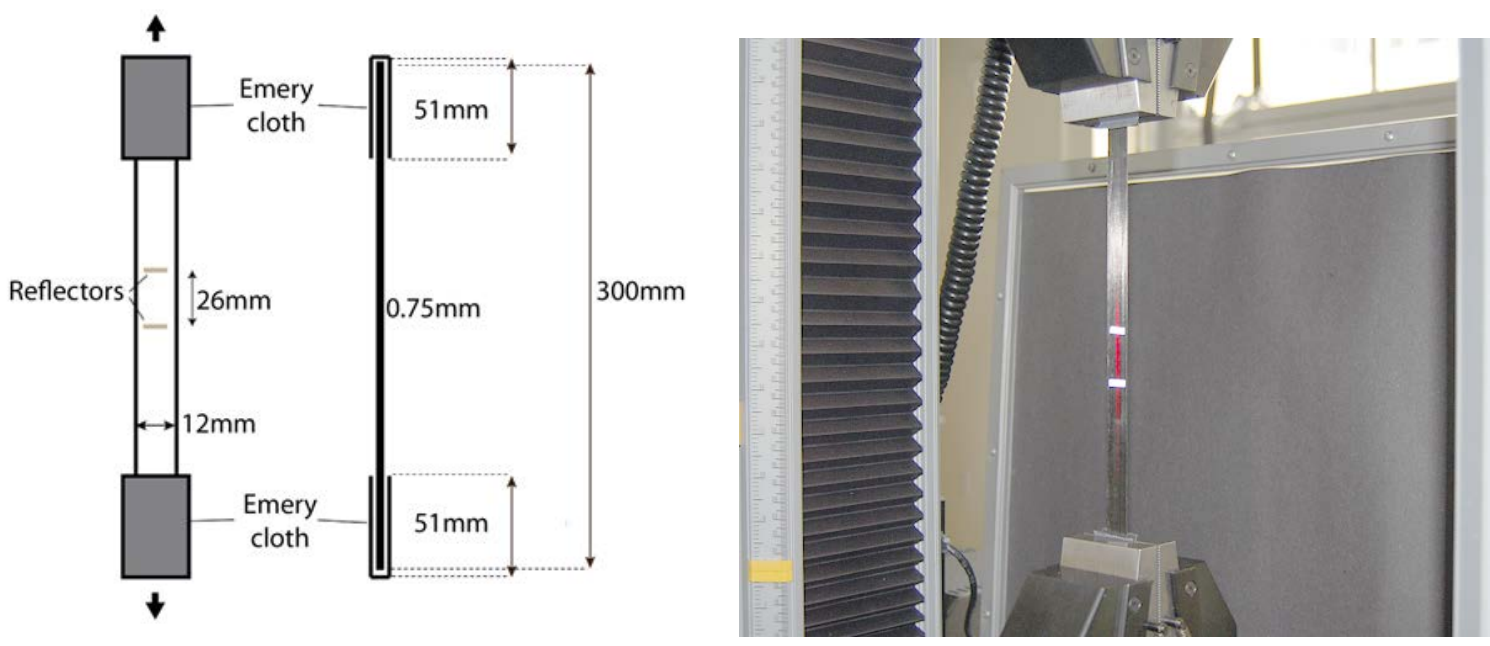

Figure 4. Tension test: schematic diagram and photo of test apparatus.

The testing machine used was an Instron 5569 with a $50 \mathrm{kN}$ load cell. The relative displacement between two reflecting strips was measured by an Epsilon LEO1 laser extensometer. The test consisted in five cycles on 3 nominally identical samples, up to a maximum strain of $0.2-0.3 \%$ at a displacement rate of $1 \mathrm{~mm} / \mathrm{min}$. It was not possible to achieve higher strains due to edge slippage.

\section{IV.B. Tension strength tests}

To prevent slippage at higher loads, single-tow samples were made in which the fibers were embedded in epoxy matrix near the ends. Aluminum tabs bonded at the end of the samples were aligned in the Instron 5569 testing machine with a $50 \mathrm{kN}$ load cell and pulled in tension. The extension of the crosshead was increased at a rate of $0.05 \mathrm{~mm} / \mathrm{s}$. 


\section{IV.C. Compression tests}

Unidirectional solid rods were chosen for these tests, because thin, flat samples have to be very short to avoid structural buckling. Moreover, if the sample is clamped at the ends in a standard compression rig the load is transmitted to the sample through shearing tractions on the end surface, and this results in a non-uniform distribution of compression stress on the cross-section of the sample.

To avoid these problems, the tips of the rods were inserted inside square aluminum caps in which two holes had been drilled to allow any voids between the rod and the cap to be filled with epoxy resin. The hole diameter was chosen so the rod would be fit snugly inside the cap. This procedure was needed as the tips of the rods need to be in complete contact with the caps, to apply the end compression as uniformly as possible.

Figure 5 shows a schematic diagram of the sample geometry, a photo of a sample taken apart to show its pieces and a picture of the sample next to a Wyoming CLC compression fixture.
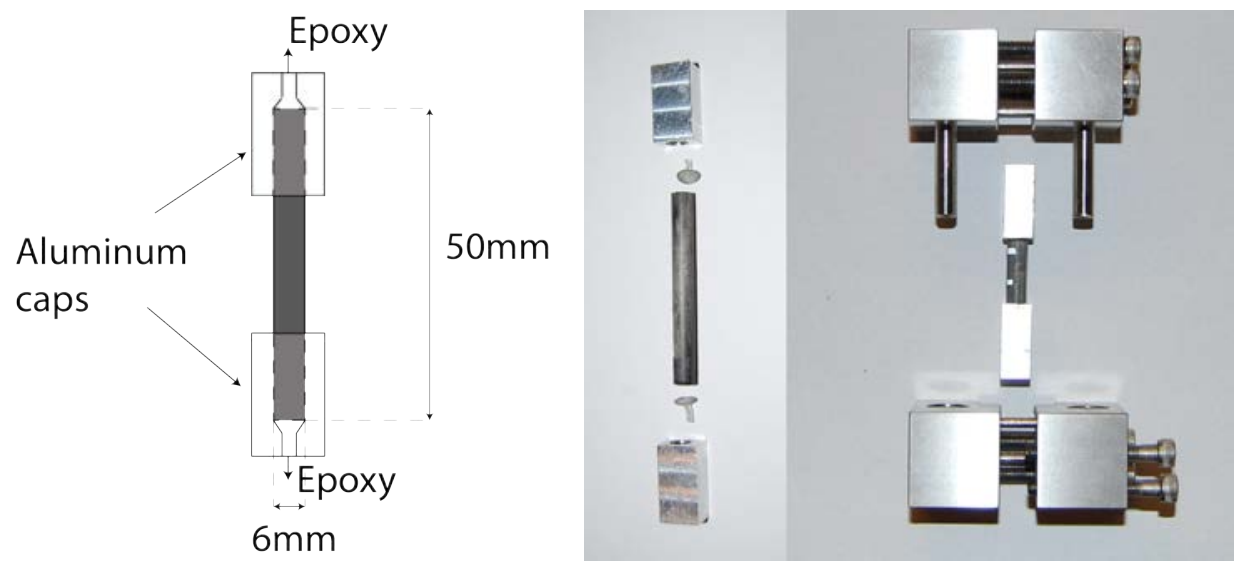

Figure 5. Compression test: schematic and photos of the sample. The first photography shows a sample after testing, with aluminum tabs and epoxy debonded purposely to describe the setup. The second photography shows a sample bonded inside between the tabs, ready to be adjusted in the Wyoming CLC compression fixture.

Once the sample had been mounted in the compression fixture, the fixture was placed between two compression platens in the Instron 5569 testing machine and tested in compression with a $1 \mathrm{kN}$ load cell, at a displacement rate of $0.05 \mathrm{~mm} / \mathrm{s}$. Since the strain at which microbuckling occurs is small, a Digital Image Correlation (DIC) system was chosen. With this method, the displacements of a speckle pattern, Figure 6 , painted on the surface of the sample are recorded with two cameras. The speckle pattern needs to be random and the speckles need to be small and dense enough to provide the required spatial resolution. After the necessary calibrations, the position of the speckles on the sample throughout the test are determined.
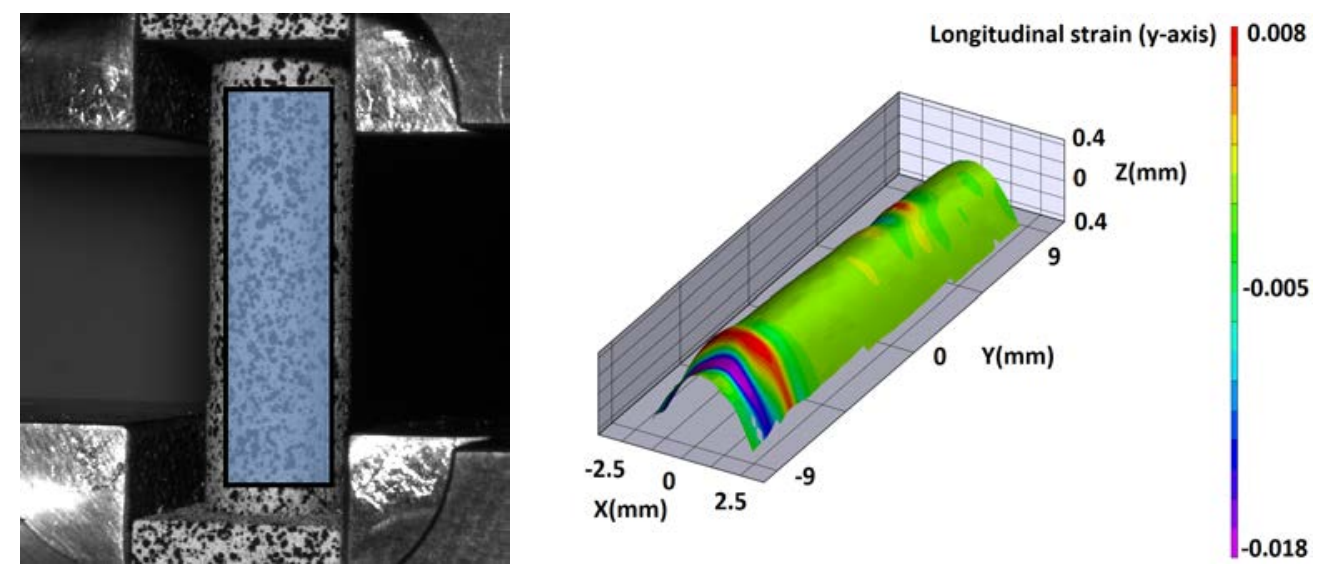

Figure 6. Sample inside the compression fixture. The area of study is highlighted in blue. The right image shows the three-dimensional shape under microbuckling of the area of study calculated with Digital Image Correlation

During each compression test, images of the sample were recorded at a speed of 2 frames/second. Those 
frames were then analyzed with VIC-3D, a DIC software from Correlated Solutions, to obtain the threedimensional displacements of the surface of the sample. Additionally, the load measured by the load cell was synchronized with the images in order to obtain the stress-strain relationship.

The area of the sample that was imaged with DIC had a length of $\sim 20 \mathrm{~mm}$ and a frontal apparent width of $\sim 5 \mathrm{~mm}$, Figure 6 . The strain in the area of study was found by defining a $15 \mathrm{~mm}$ long virtual extensometer in the longitudinal direction. It was found that averaging the longitudinal strain over the whole area of study would yield similar results.

\section{IV.D. Bending tests}

Four-point bending tests were carried out, based on the ASTM D6272 standard. The setup and geometry for these tests, on unidirectional flat samples as detailed in Table 2, are shown in Fig. 7. The span between the outer supports was $176 \mathrm{~mm}$ and the distance between the inner load points $50 \mathrm{~mm}$. This relatively long span was chosen to minimize shearing near the points of application of the loads, by limiting the force that would need to be applied. The moment-curvature relationship for the sample can be easily obtained from the force applied by the Instron 5569 testing machine and the displacement between two reflecting strips attached to the sample and the upper part of the fixture, measured with a laser extensometer.
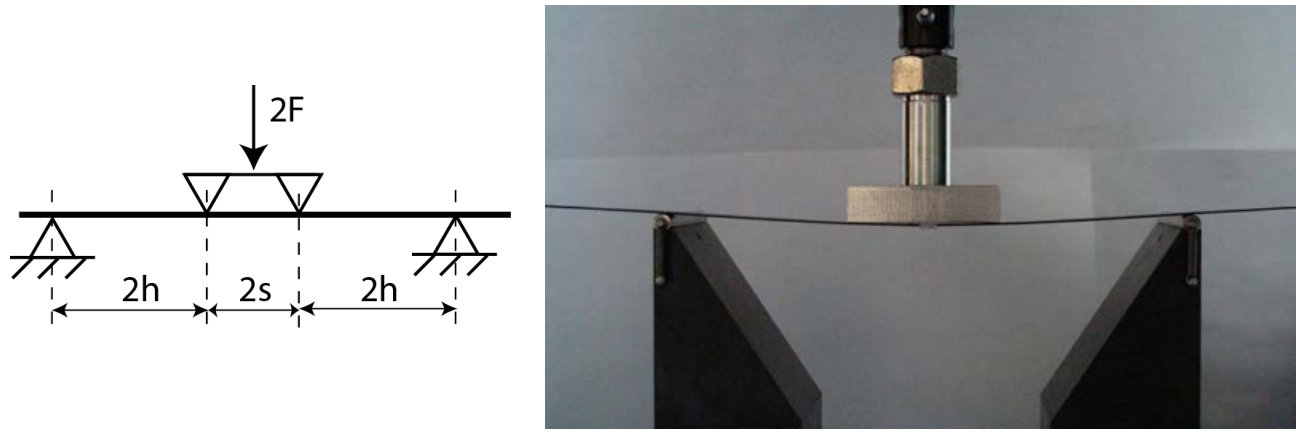

Figure 7. Bending test: schematics and photo. The bending fixtures have been chosen and adjusted to achieve the maximum span possible to minimize the shearing force and avoid kinking. $h=73 \mathbf{m m}, s=25 \mathrm{~mm}$.

\section{Test Results}

This section presents the test results that were obtained with the techniques outlined in the previous section.

\section{V.A. Tension}

Figure 8 shows the stress-strain relationship obtained from a set of 5 load-unload cycles on 3 samples. The extensometer measurements were smoothed using a 500-point moving average. At strains of less that $0.1 \%$ all samples showed some non-linearity, which is attributed to initial slackness and twisting of the sample. The modulus of each sample has been estimated by a least squares linear fit for strains higher than $0.1 \%$ and the resulting values are presented in Table 3 .

These results are in close agreement with the theoretical estimate obtained from the rule of mixtures, which gave a value coinciding with the measured modulus of the third sample and around $12 \%$ larger than those of the other two.

\begin{tabular}{|l|c|c|c|c|}
\hline & Sample 1 & Sample 2 & Sample 3 & Theoretical estimate \\
\hline$E_{1}(\mathrm{GPa})$ & 69 & 68 & 78 & 78 \\
\hline
\end{tabular}

Table 3. Longitudinal tensile stiffness.

The measurements made from the second set of tension samples, which were tested up to failure, are presented in Figure 9. The overall behavior was quite repeatable, with all samples showing a small amount 


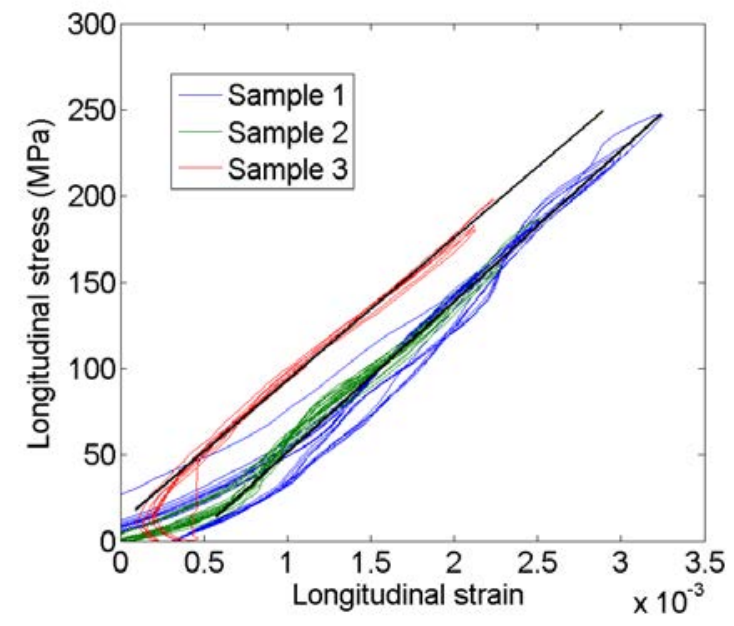

Figure 8. Tensile behavior at small strains.

of initial non-linearity and failure strengths in the range 2200-2500 MPa. The test results are summarized in Table 4.

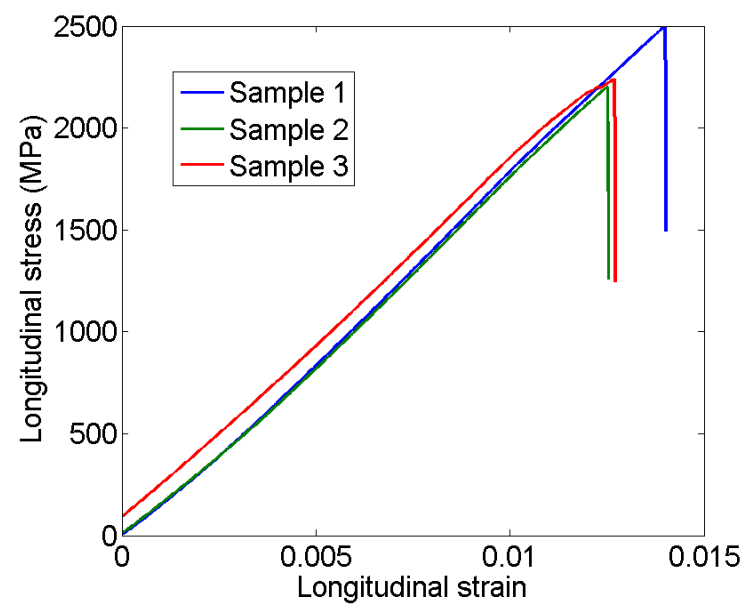

Figure 9. Tensile behavior up to failure.

\begin{tabular}{|l|c|c|c|c|c|}
\hline & Sample 1 & Sample 2 & Sample 3 & Fiber strength & Bundle strength \\
\hline Tensile strength $(\mathrm{GPa})$ & 2.2 & 2.2 & 2.5 & 4.3 & 1.5 \\
\hline
\end{tabular}

Table 4. Measured tensile strength and comparison with single fiber strength and estimated bundle strength with Monte Carlo simulation.

The bundle strength estimate from Section III.B is on the order of $30 \%$ smaller than the measured strength, indicating that the simple model used to determine bundle failure provides realistic, but somewhat over-conservative estimates. The discrepancy is likely due to the fact that the Weibull distribution parameters in Ref., ${ }^{6}$ used for the present estimate, were obtained from a batch of about $10020 \mathrm{~mm}$ long carbon fibers. A larger set of longer fibers should be used to avoid extrapolating across 2 orders of magnitude in the number of fibers and one order of magnitude in length. 


\section{V.B. Compression}

Figure 10 shows stress-strain plots for two nominally identical samples. The plot on the left was obtained from a single $15 \mathrm{~mm}$ long virtual extensometer defined on the surface of the sample, whereas the plot on the right was obtained from the average longitudinal strain over the region imaged by DIC. The difference is a small change in the average slopes.
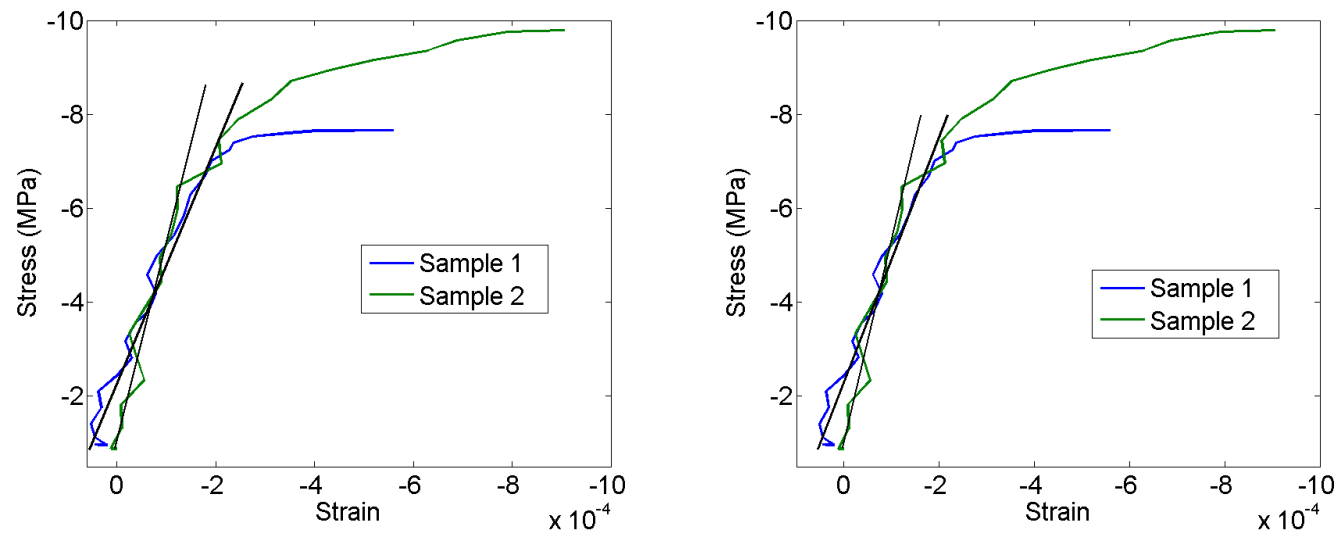

Figure 10. Compressive stress-strain curves for two different samples.

The compressive stiffness has been calculated from the slope of the steepest part of the stress-strain curves, to obtain an average of $34 \mathrm{GPa}$. This value is about three times smaller than the rule of mixtures estimate of $108 \mathrm{GPa}$. It is believed that a likely cause of this discrepancy is initial fiber waviness in the material, see Figure 11. Despite the efforts made to ensure a uniform loading of the compression samples, non-uniformity of the stress distribution may also be responsible for the low modulus measurements.

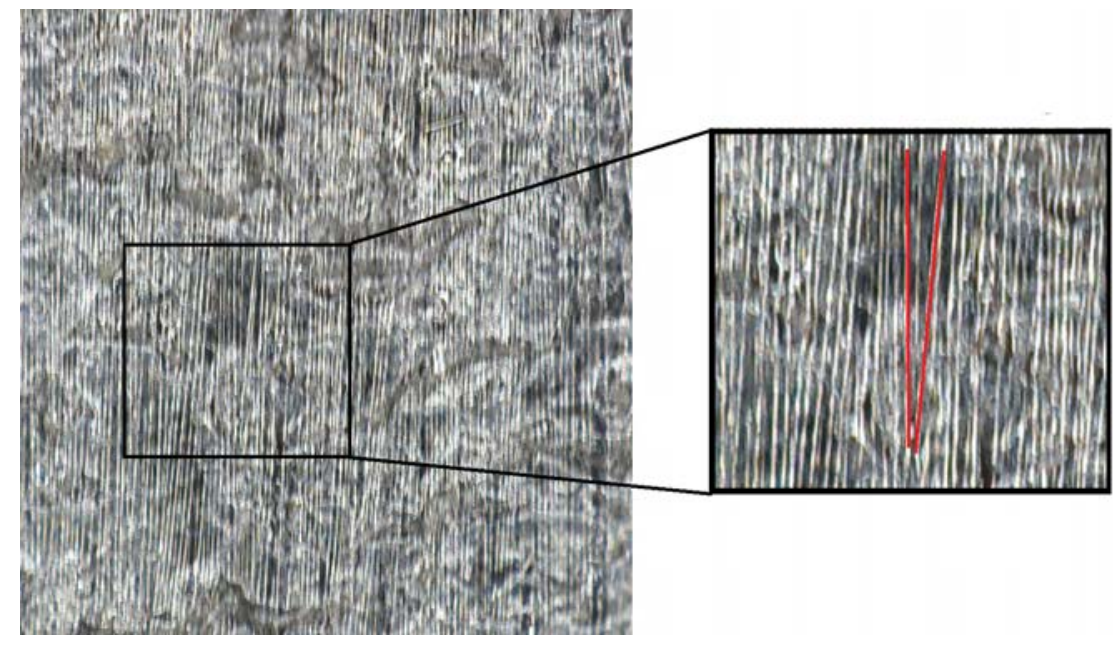

Figure 11. Optical microscope image of fiber distribution on the surface of a sample. The enlarged image shows that some fibers are not parallel to the longitudinal direction.

The plateau in the measured stress-strain behavior corresponds to the onset of fiber microbuckling, which in Section III.C was estimated to occur at a critical stress of $\sigma_{c r i t}=5.7 \mathrm{MPa}$. The measured values were about $35 \%$ larger.

Figure 12 shows plots of circumferential vs. longitudinal strains in the compression samples, from which the Poisson's ratio $\nu_{12}$ has been measured. The measured values are in the range 0.02 to 0.06 , which are between 5 and 15 times smaller than the value of 0.34 estimated with the rule of mixtures. However, it should be noted that a numerical homogenization using a finite element model that will be presented elsewhere has given $\nu_{12}=0.14$, which suggests that using the rule of mixtures is not a good way to estimate the Poisson's ratio for this material. 


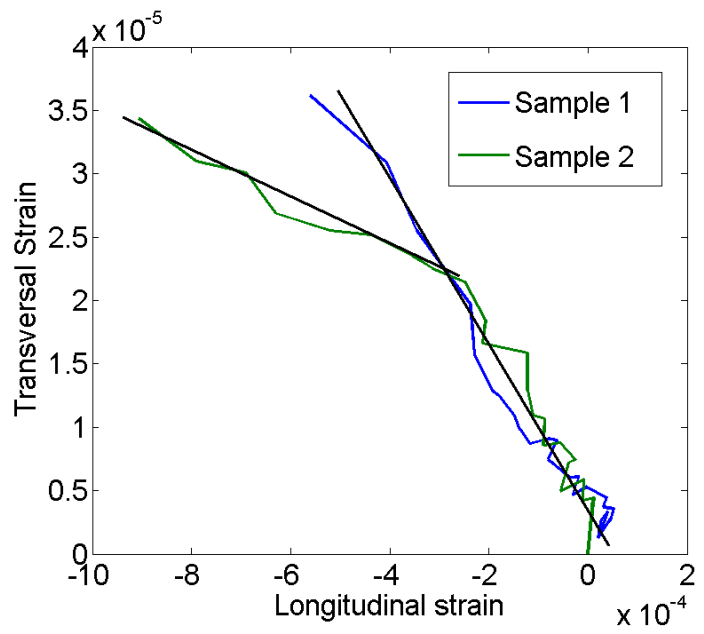

Figure 12. Longitudinal strain vs. circumferential strain on surface of compression samples.

Table 5 summarizes the stiffness and critical stress measured on the two samples, and compares them with the analytical estimates from Section III.

\begin{tabular}{|c|c|c|c|c|c|c|}
\hline Sample & $E_{1}(\mathrm{MPa})$ & $E_{1, t h}(\mathrm{MPa})$ & $\sigma_{\text {crit }}(\mathrm{MPa})$ & $\sigma_{\text {crit }, \text { th }}(\mathrm{MPa})$ & $\nu_{12}$ & $\nu_{12, t h}$ \\
\hline 1 & 27 & 108 & 7.5 & 5.7 & 0.06 & 0.34 \\
\hline 2 & 40 & 108 & 8.0 & 5.7 & $0.02 \sim 0.06$ & 0.34 \\
\hline
\end{tabular}

Table 5. Values of compression stiffness, critical stress and Poisson's ratio.

\section{V.C. Bending}

Figure 13 shows the measured moment-curvature behavior of two test samples. The first load-unload cycle shows an initial linear response upon loading, reaching a plateau that is believed to be due to fiber microbuckling, followed by hysteretic behavior upon unloading. The behavior of both samples was softer during the second and third cycles, as already observed in previous tests by Lopez and Pellegrino. ${ }^{2}$ The moment-curvature relation for the second sample has a negative slope for moments of less than $0.02 \mathrm{Nm}$, due to initial out-of-flatness of this sample.
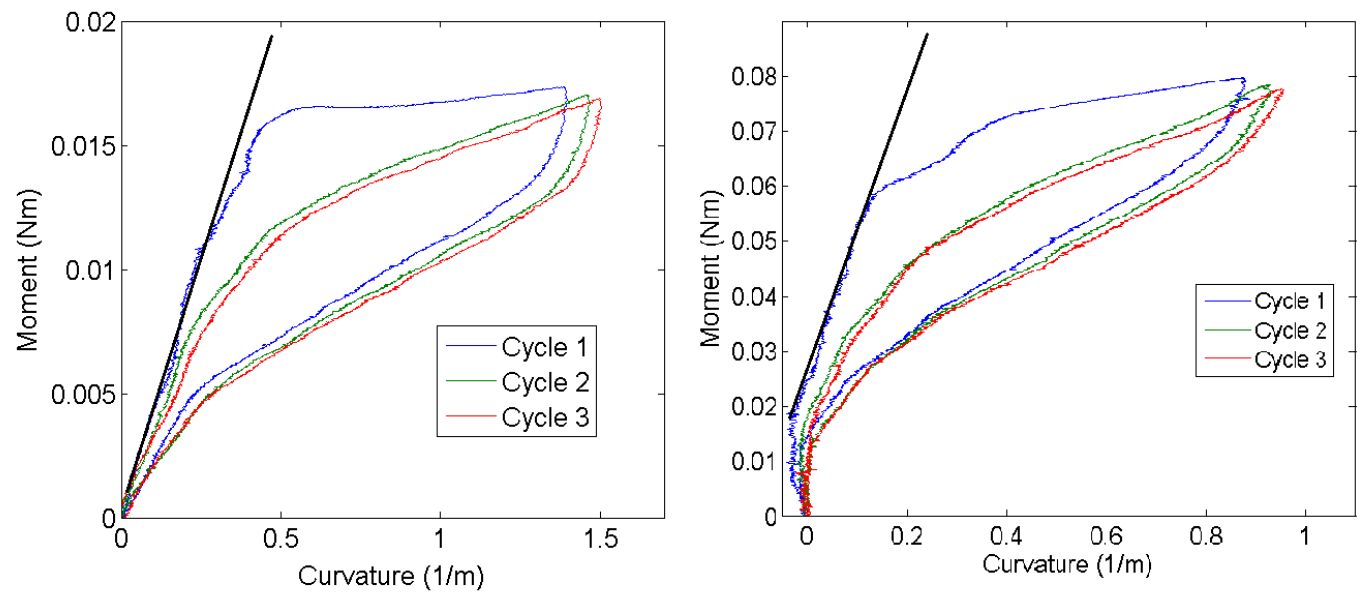

Figure 13. Moment-curvature plots for 3 load-unload cycles on samples with thickness of $t=0.75 \mathrm{~mm}$ and $t=1.5 \mathrm{~mm}$. The straight lines define the bending stiffness during the first cycle, before fiber microbuckling.

The stiffness of these samples was obtained from the slope of the linear part of the loading curve. The 
bending stiffness is calculated from

$$
E I=\Delta M / \Delta \kappa
$$

Dividing the measured bending stiffness by the second moment of area of each sample we obtain the modulus, which is compared in Table 6 to the rule of mixtures estimates from Section III. The difference is in the range $\pm 7 \%$.

\begin{tabular}{|c|c|c|c|c|c|c|}
\hline Sample & $t(\mathrm{~mm})$ & $V_{f}$ & $E_{1}$ & $E_{1, t h}(\mathrm{MPa})$ & $M_{\text {crit }}(\mathrm{Nm})$ & $M_{\text {crit }, \text { th }}(\mathrm{Nm})$ \\
\hline Thin & 0.75 & 33 & 83 & 78 & 0.017 & 0.005 \\
\hline Thick & 1.5 & 35 & 77 & 83 & 0.06 & 0.023 \\
\hline
\end{tabular}

Table 6. Stiffness and critical moment of bending samples.

The softening that marks the end of the approximately linear part in the moment-curvature plots corresponds to the onset of fiber microbuckling in the regions of highest compressive stress. The critical moment at which this happens can be calculated from the fiber microbuckling stress $\sigma_{\text {crit }}$ estimated previously:

$$
M_{c r i t, t h}=\frac{\sigma_{c r i t} I}{y_{\max }}
$$

Theoretical estimates obtained from Equation 7 have been presented in the last column of Table 6 and it can be seen that these estimates are about three times smaller than the measured critical moments. This result suggests that the fiber microbuckling stress under bending is about three times larger than for pure compression, which is the condition assumed in the estimation of $\sigma_{\text {crit }}$.

\section{Conclusion}

The experimental characterization of the L'Garde high strain material has posed a number of challenges, which have been addressed in the present study by introducing modifications to standard test methods, such as using end caps to introduce a uniform compression stress or using very thin bending samples and paying great attention to the alinement of the 4-point bending rig, to avoid inducing premature fiber microbuckling and kinking in the sample.

Once such effects had been minimized, several aspects of the experimentally observed behavior have been found to match our expectations, based on simple rule-of-mixtures estimates of stiffness, Weibull distribution of fiber strength, and elastic fiber microbuckling.

Tension and bending tests have yielded longitudinal modulus values comparable to the estimate provided by the rule of mixtures. However, this estimate appears not to work for compression. Perhaps the rule of mixtures does not represent well the behavior of the material under compression or, more likely, fiber waviness or other defects in the material need to be taken into account or minimized during fabrication of the samples. The possibility of errors introduced by the experimental technique cannot be discarded either. The measured values of the Poisson's ratio, obtained from compression tests, also do not agree with the rule of mixtures estimates. Further measurements of the Poisson's ratio should be made under tension, and alternative estimate techniques will need to be investigated.

Tensile samples containing stiff epoxy near the ends allowed us to measure the tensile failure strength. The transition zones between soft matrix and epoxy did not weaken the samples near the ends, as all samples failed close to the center and far from the transition. The estimated strength of the fiber bundle calculated with the Monte-Carlo simulation using the Weibull distribution for probability of failure was realistic although over-conservative, and much more accurate than estimates based on the failure stress of a single fiber.

Estimates based on fiber microbuckling of the plateau stress, for compression, and of the softening moment, for bending, were found to be conservative. For pure compression, the estimated strength was of the same order than the measured strength, and could be used as a useful lower bound for design. However, for case of bending the estimated values are much too small to justify this approach. 


\section{Acknowledgements}

The material fabrication and characterization study presented in this paper was funded by NASA JPL under the SBIR-Phase 1 "Thermally-Stable High Strain Deployable Structures". The material development and manufacture of the Miura-ori composite lamina shown in Figure 1 were done under internal R\&D funded by L'Garde, Inc. IM thanks the Fulbright Foundation for financial support.

\section{References}

${ }^{1}$ D. Campbell, M.S. Lake, and K. Mallick. A study of the bending mechanics of elastic memory composites, 45th AIAA/ASME/ASCE/AHS/ASC Structures, Structural Dynamics, and Materials Conference, Palm Springs, CA, April 1922, AIAA-2004-1636.

${ }^{2}$ F. Lopez Jimenez and S. Pellegrino. Folding of fiber composites with a hyperelastic matrix. International Journal of Solids and Structures 49, February 2012(3-4): 395-407.

${ }^{3}$ T.W. Murphey, T. Meink, and M.M. Mikulas. Some micromechanics considerations of the folding of rigidizable composite materials. 42nd AIAA/ASME/ASCE/AHS/ASC Structures, Structural Dynamics, and Materials Conference, AIAA-20011418.

${ }^{4}$ J.M. Mejia-Ariza, K. Guidanean, T.M. Murphey and A. Biskner. Mechanical characterization of L'Garde elastomeric resin composite materials. 51st AIAA/ASME/ASCE/AHS/ASC Structures, Structural Dynamics, and Materials Conference, AIAA-2010-2701, 2010.

${ }^{5}$ J.B. Donnet, T.K. Wang, J.C.M. Peng and S. Rebouillat. Carbon Fibers, Marcel Dekker, New York, third edition, 1998.

${ }^{6}$ F. Lopez Jimenez and S. Pellegrino. Failure of carbon fibers at a crease in a fiber-reinforced silicone sheet. Accepted for publication in Journal of Applied Mechanics.

${ }^{7}$ B.W. Rosen. Fiber Composite Materials, Metals Park, Ohio, 1965. 